\title{
Pricing rule based on non-arbitrage arguments for random volatility and volatility smile
}

\author{
N.G.Dokuchaev* \\ Department of Mathematics and Computer Science, The University of West Indies, Mona \\ Jamaica
}

October 27, 2018

\begin{abstract}
We consider a generic market model with a single stock and with random volatility. We assume that there is a number of tradable options for that stock with different strike prices. The paper states the problem of finding a pricing rule that gives BlackScholes price for at-money options and such that the market is arbitrage free for any number of tradable options, even if there are two Brownian motions only: one drives the stock price, the other drives the volatility process. This problem is reduced to solving a parabolic equation.
\end{abstract}

Key words: diffusion market model, stochastic volaltility, arbitrage free model

\section{Introduction}

We consider a market model with a single stock and with random volatility. Most practitioners have adapted the famous Black-Scholes model as the premier model for pricing and hedging of options. This model consists of two assets: the risk free bond or bank account and the risky stock. It is assumed that the dynamics of the stock is given by a random process with some standard deviation of the stock returns (the volatility coefficient, or volatility). The dynamics of bonds is deterministic and exponentially increasing

\footnotetext{
${ }^{*}$ Corresponding address: Department of Mathematics and Computer Science, The University of West Indies, Mona, Kingston 7, Jamaica W.I. Email: ndokuch@uwimona.edu.jm
} 
with a given risk-free rate. In the classic Black-Scholes model, the volatility is assumed to be given and fixed. However, empirical research shows that the real volatility is timevarying and random. Moreover, it is commonly recognized that Black-Scholes formula gives unbiased estimation for at-money options only, and it gives a systematic error for in-money and out-of-money options; in fact, that means that there is a gap between historical and implied volatility that generates so-called volatility smile for the implied volatility; see e.g. Black and Scholes (1972), Day and Levis (1992), Derman et al. (1996), Hauser and Lauterbach (1997), Taylor and Xu (1994). A very detailed review can be found in Mayhew (1995)). Many authors emphasize that the main difficulty in modifying the Black-Scholes and Merton models is taking into account this fact.

To fill this gap, a number of deterministic and stochastic equations for volatility were proposed (see e.g. Christie (1982), Johnson and Shanno (1987), Hull and White (1987), Masi et al. (1994) and more recent papers in Jarrow (ed.) (1998). In some other approach, a special temporal scale is used to find the time when historical volatility coincides with implied volatility (see e.g. Geman and Ane (1996)).

This paper suggests some another approach to the problem based on non-arbitrage arguments (see, e.g., Harrison and Pliska (1981) and Jouini (1996)). We take a very generic model for the random volatility process (more precisely, for historical volatility process), and then look for a pricing rule that

- gives Black-Scholes price for at-money options;

- ensures that arbitrage possibilities are absent for an extended market that includes a number of tradable options for that stock with different strike prices, and such that there are two Brownian motions only: one drives the stock price, the other drives the volatility process.

Notice that similar non-arbitrage arguments are used traditionally for bond pricing; for the generic model, there is only one Brownian motions driving the interest rate there are many bonds. We explore the same approach for multi-option market to find a model for the volatility smile.

We show below the required pricing rule exists if some boundary value problem for a parabolic equation is solvable; the price is expressed via solution of this equation. This 
boundary value problem looks simple, but, unfortunately, it appears to be difficult to solve; the boundary conditions cannot be reduced to known ones. Most part of the paper devoted analysis of solvability of this boundary value problem. We obtained some existence and uniqueness theorem and prior estimations for the solution; we found a also formula for the Fourier transform of the solution via expectation of a integral functional of the volatility process.

\section{Definitions}

Consider the diffusion model of a securities market consisting of a risk free bond or bank account with the price $B(t), t \geq 0$, and a risky stock with price $S(t), t \geq 0$. The prices of the stocks evolve according to the following stochastic differential equation

$$
d S(t)=S(t)(a(t) d t+\sigma(t) d w(t)), \quad t>0,
$$

where $w(t)$ is a standard Wiener process, $a(t)$ is an appreciation rate, $\sigma(t)$ is a volatility coefficient. The initial price $S(0)>0$ is a given deterministic constant. The price of the bond evolves according to the following equation

$$
B(t)=e^{r t} B(0)
$$

where $r \geq 0$ and $B(0)$ are given constants.

Set $v(t) \triangleq \sigma(t)^{2}$. We assume that the process for $v(t)$ evolves as

$$
d v(t)=v(t)(\widehat{a} d t+\widehat{\sigma} d \widehat{w}(t)), \quad t>0
$$

where $\widehat{a}$ and $\widehat{\sigma} \neq 0$ are known constants, and $\widehat{w}(\cdot)$ is a Wiener process independent of $w(\cdot)$. The initial volatility $v(0)>0$ is a given deterministic constant. Moreover, we assume for the sake of simplicity that the volatility process has zero drift, i.e. $\widehat{a}=0$ (an extension for the case $\widehat{a} \neq 0$ does requires principal changes and is rather technical).

We assume that $(w(\cdot), \widehat{w}(\cdot))$ is a standard Wiener process on a given standard probability space $(\Omega, \mathcal{F}, \mathbf{P})$, where $\Omega$ is a set of elementary events, $\mathcal{F}$ is a complete $\sigma$-algebra of events, and $\mathbf{P}$ is a probability measure.

Further, we assume that there are available European options on that stocks with the same expiration time $T$ and different strike prices $K \in \mathcal{K}$, where $\mathcal{K} \subset(0,+\infty)$ is a given 
set. For simplicity, we assume that the set $\mathcal{K}$ is finite, i.e. $\mathcal{K}=\left\{K_{1}, \ldots, K_{N}\right\}$, where $N$ is an integer, possibly a large number. (Generalization for the case of infinite number of $K$ and different expiration times is rather technical).

We shall denote $P_{c}(t, K)$ and $P_{p}(t, K)$ the prices for the call option with the claim $(S(T)-K)^{+}$and for the put option with the claim $(K-S(T))^{+}$respectively, where $T$ is the expiration time, $K$ is the strike price. We shall consider options as additional tradable assets, i.e. we shall consider bond-stock-options market. Under our assumptions, the market is incomplete (i.e. options can not be replicated). We shall look for a reasonable model for prices $\left\{P_{c}(t, K), P_{p}(t, K)\right\}_{K \in \mathcal{K}}$ (i.e. a reasonable pricing rule); the main requirement is that the market must be arbitrage free. Since we have only two driving Brownian motions $w(\cdot)$ and $\widehat{w}(\cdot)$, we need to prevent arbitrage possibilities for the case of large $N$.

Let $H_{B S, c}(x, v, t, K)$ and $H_{B S, p}(x, v, t, K)$ denotes Black-Scholes prices for the put and call options with the claims $(S(T)-K)^{+}$and $(K-S(T))^{+}$respectively given condition $S(t)=x,\left.\sigma(s)^{2}\right|_{[t, T]} \equiv v$. (Note that $\mathbf{E}\left\{\sigma(s)^{2} \mid v(t)=v\right\}$ is $v$ for any $s \geq t$.) More precisely,

$$
\begin{aligned}
\tilde{H}_{B S, c}(x, v, t, \tilde{K}) & =x \Phi\left(d_{+}(x, v, t, \tilde{K})\right)-\tilde{K} \Phi\left(d_{+}(x, v, t, \tilde{K})\right), \\
\tilde{H}_{B S, p}(x, v, t, \tilde{K}) & =\tilde{H}_{B S, 1}(x, v, t, \tilde{K})+x-\tilde{K}
\end{aligned}
$$

where

$$
\begin{aligned}
& \Phi(x) \triangleq \frac{1}{\sqrt{2 \pi}} \int_{-\infty}^{x} e^{-\frac{t^{2}}{2}} d t \\
& d_{+}(x, v, t, \tilde{K}) \triangleq \frac{\log x-\log \tilde{K}}{\sqrt{(T-t) v}}+\frac{\sqrt{(T-t) v}}{2}, \quad d_{-}(x, v, t, \tilde{K}) \triangleq \frac{\log x-\log \tilde{K}}{\sqrt{(T-t) v}}-\frac{\sqrt{(T-t) v}}{2}
\end{aligned}
$$

To make the following definitions more compact, we shall denote

$$
\begin{aligned}
& P_{1}(t, K) \triangleq P_{c}(t, K), \quad P_{2}(t, K) \triangleq P_{p}(t, K) \\
& H_{B S, 1}(x, v, t, K) \triangleq H_{B S, c}(x, v, t, K), \quad H_{B S, 2}(x, v, t, K) \triangleq H_{B S, p}(x, v, t, K) .
\end{aligned}
$$

Definition 2.1 A process $v_{K}(t)$ is said to be implied volatility given the strike price $K$ and the type of option (i.e. put or call), if $P_{j}(t, K) \equiv H_{B S, j}\left(t, S(t), v_{K, j}(t), K\right), j=1,2$.

Clearly, the problem of finding prices $\left\{P_{c}(t, K), P_{p}(t, K)\right\}_{K \in \mathcal{K}}$ can be reformulated as the problem of finding implied volatilities $\left\{v_{K, j}(t)\right\}_{K \in \mathcal{K}, i=1,2}$. 
As known, if $v$ is non-random, i.e. if $\widehat{\sigma}=0$, then the market with Black-Scholes

prices $P_{j}(t, K) \triangleq H_{B S, j}(t, S(t), v(t), K)$ is arbitrage free (i.e. the market is arbitrage free with $v_{K}(t) \equiv v$, when the implied volatility and the historical volatility are equal). Unfortunately, for random volatilites, this pricing rule leads to a model with arbitrage possibilities.

Proposition 2.1 If $\widehat{\sigma} \neq 0$ then the pricing rule $P_{j}(t, K) \triangleq H_{B S, j}(t, S(t), v(t), K)$ does not allow arbitrge for $N=1$ and allows arbitrage for $N \geq 2$.

One of possible ways to construct an arbitrage free market is to include additional Brownian motions for the equations for the implied volatilities $v_{K, j}(t)$. (Remind that the system $\left\{v_{K, j}(t)\right\}$ defines prices as $\left.P_{j}(t, K)=H_{B S, j}\left(t, S(t), v_{K}(t), K\right)\right)$. For example, the model will be arbitrage free if

$$
d v_{K, j}(t)=v_{K, j}(t)\left(\widehat{a}_{K, j} d t+\widehat{\sigma}_{K, j} d \widehat{w}(t)+\sigma_{K, j} d w_{K, j}(t)\right), \quad t>0, \quad j=1,2,
$$

where $\left\{w_{K, j}(\cdot)\right\}_{K \in \mathcal{K}, j=1,2}$ is a system of independent Brownian motions that does not depend on $(w(\cdot), \widehat{w}(\cdot))$, and where $\widehat{\sigma}(K, j), \sigma_{K, j} \neq 0$ are constants.

We introduce here another arbitrage free market model with only two driving Brownian motions and arbitrarily large $N>0$.

Set

$$
\tilde{S}(t) \triangleq e^{-r t} S(t), \quad \tilde{K} \triangleq e^{-r T} K, \quad \tilde{P}(t, \tilde{K}) \triangleq e^{-r t} P(t, K), \quad K \in \mathcal{K} .
$$

Let $\tilde{\mathcal{K}} \triangleq\left\{\tilde{K}=e^{-r T} K: K \in \mathcal{K}\right\}$. Let the function $\tilde{H}_{B S}(\cdot): Q \times \tilde{\mathcal{K}} \rightarrow \mathbf{R}$ be such that $e^{r t} \tilde{H}_{B S, j}(x, v, t, \tilde{K}) \equiv H_{B S, j}\left(e^{r T} x, v, t, K\right)$.

Let $\mathbf{P}_{*}$ be the risk-neutral measure such that the process $w_{*}(t) \triangleq \int_{0}^{t} \sigma(s)^{-1}(a(s)-$ $r) d s+w(t)$ is condionally a Wiener process given $\sigma(\cdot)$. Let $\mathbf{E}_{*}$ be the corresponding expectation. As is known, $\tilde{S}(t)$ is a martingale under $\mathbf{P}_{*}$, and $\mathbf{E}_{*}\{S(T) \mid S(t)\}=e^{r(T-t)} S(t)$. Thus, it is natural to say that an option with the strike price $K$ is at-money at time $t$ if $e^{r(T-t)} S(t)=K$, i.e. $e^{r(T-t)} e^{r t} \tilde{S}(t)=K$, or $\tilde{S}(t)=\tilde{K}$.

Let $\mathcal{F}_{t}$ be the filtration generates by $(S(t), v(t))$.

Set $\widehat{P}_{j}(t, x, \tilde{K}) \triangleq \mathbf{E}\left\{\tilde{P}_{j}(t, \tilde{K}) \mid \tilde{S}(t)=x\right\}$.

We are looking for the set of prices $\left\{P_{j}(t, K)\right\}_{K \in \mathcal{K}, j=1,2}$ or $\left\{\tilde{P}_{j}(t, \tilde{K})\right\}_{\tilde{K} \in \mathcal{K}, j=1,2}$ such that the following conditions are satisfied: 
(A1) Processes $P_{j}(t, K)$ and $v_{K, j}(t)$ are $\mathcal{F}_{t}$-adapted, i.e. the only source of randomness in the model is the pair of Brownian motions $(w(\cdot), \widehat{w}(\cdot))$;

(A2) The market is arbitrage free (even if the number of tradable options $N$ is arbitrarily large);

(A3) For at-money options, when $\tilde{S}(t) \sim \tilde{K}$, the price is close to the Black-Scholes price; more precisely,

$$
\begin{aligned}
& \widehat{P}_{j}(t, \tilde{K}, \tilde{K}) \equiv \tilde{H}_{B S, j}(\tilde{K}, v(t), t, \tilde{K}) \\
& \left.\left.\frac{\partial \widehat{P}_{j}}{\partial x}(t, x, \tilde{K})\right|_{x=\tilde{K}} \equiv \frac{\partial \tilde{H}_{B S, j}}{\partial x}(x, v(t), t, \tilde{K})\right|_{x=\tilde{K}}
\end{aligned}
$$

In particular, if $\tilde{S}(t)=\tilde{K}$, then the implied volatility and the historical volatility are equal, i.e. $v_{K, j}(t) \equiv v(t)$.

(A4) There exists a sequence of Markov times $\left\{T_{k}\right\}_{k=1}^{+\infty}$ with respect for the filtration $\mathcal{F}_{t}$ such that $T_{k} \rightarrow T$ a.s., $\mathbf{E} \int_{0}^{T_{k}} P_{j}\left(t, K_{i}\right)^{2} d t<+\infty$, and there exists Ito's differential $d_{t} P_{j}(t, K), t \in\left(0, T_{k}\right)$, for all $k$.

\section{Strategies for bond-stock-options market}

Let $X(0)>0$ be the initial wealth at time $t=0$ and let $X(t)$ be the wealth at time $t>0$. We assume that the wealth $X(t)$ at time $t \geq 0$ is

$$
X(t)=\beta(t) B(t)+\gamma_{0}(t) S(t)+\sum_{j=1}^{2} \sum_{i=1}^{N} \gamma_{i, j}(t) P_{j}\left(t, K_{i}\right)
$$

Here $\beta(t)$ is the quantity of the bond portfolio, $\gamma_{0}(t)$ is the quantity of the stock portfolio, $\gamma_{i, j}(t)$ is the quantity of the options portfolio with the strike price $K_{i}$ (put for $j=1$ and call for $j=2)$, and $\gamma(t)=\left(\gamma_{0}(t), \gamma_{1,1}(t), \ldots, \gamma_{N, 1}(t), \gamma_{1,2}(t), \ldots, \gamma_{N, 2}(t)\right), t \geq 0$. The pair $(\beta(t), \gamma(t))$ describes the state of the bond-stocks securities portfolio at time $t$. Each of these pairs is called a strategy.

The process $\tilde{X}(t) \triangleq e^{-r t} X(t)$ is said to be the normalized wealth.

Definition 2.2 A pair $(\beta(\cdot), \gamma(\cdot))$ is said to be an admissible strategy if $\beta(t), \gamma_{i, j}(t)$, $j=1,2, i=0,1, \ldots, N$, are random processes which are progressively measurable with 
respect to the filtration $\mathcal{F}_{t}$ and such that there exists a sequence of Markov times $\left\{T_{k}\right\}_{k=1}^{+\infty}$ with respect for the filtration $\mathcal{F}_{t}$ such that $T_{k} \rightarrow T$ a.s. and

$$
\mathbf{E} \int_{0}^{T_{k}}\left(\beta(t)^{2} d t+S(t)^{2} \gamma_{0}(t)^{2}+\sum_{j=1}^{2} \sum_{i=1}^{N} P_{j}\left(t, K_{i}\right)^{2} \gamma_{i}(t)^{2}\right) d t<+\infty \quad \forall k .
$$

Note that more simple static options strategies were considered in Dokuchaev (2002), Ch.3.

Definition 2.3 A pair $(\beta(\cdot), \gamma(\cdot))$ is said to be an admissible self-financing strategy, if

$$
d X(t)=\beta(t) d B(t)+\gamma_{0}(t) d S(t)+\sum_{j=1}^{2} \sum_{i=1}^{N} \gamma_{i} d_{t} P_{j}\left(t, K_{i}\right)
$$

In fact, (2.7) is equivalent to

$$
d \tilde{X}(t)=\gamma_{0}(t) d \tilde{S}(t)+\sum_{j=1}^{2} \sum_{i=1}^{N} \gamma_{i}(t) d_{t} \tilde{P}_{j}\left(t, \tilde{K}_{i}\right)
$$

(It can be shown, for example, similarly Dokuchaev and Zhou (2001), where the same equation was derived bond-stock market).

Let $\mathbf{D} \subseteq \mathbf{R}^{m}$ be any domain. Let $\mathbf{r}(\cdot): \mathbf{D} \rightarrow \mathbf{R}$ be a measurable function such that $\bar{r}(x) \geq 0(\forall x)$. Consider the Hilbert space $L_{2}(\mathbf{D}, \mathbf{r})$ with the weight $\mathbf{r}(\cdot)$ that consist of functions $f: \mathbf{D} \rightarrow \mathbf{R}$ such that $\|f(\cdot)\|_{L_{2}(\mathbf{D}, \mathbf{r})} \triangleq\left(\int_{\mathbf{D}}|f(x)|^{2} \mathbf{r}(x) d x\right)^{1 / 2}<+\infty$. Consider the Sobolev weighted spaces $W_{2}^{k}(\mathbf{D}, \mathbf{r})$ with the weight $\mathbf{r}(\cdot)$ that consist of functions $f(\cdot)$ such that $\sum_{l=0}^{k}\left\|\frac{d^{l} f}{d x^{l}}(\cdot)\right\|_{L_{2}(\mathbf{D}, \mathbf{r})}<+\infty$.

Let $\widehat{r}(z, y) \triangleq \frac{1}{1+e^{3 y}}$. Let $\mathcal{H} \triangleq L_{2}\left(\mathbf{R}^{2}, \widehat{r}\right), \mathcal{H}^{1} \triangleq W_{2}^{1}\left(\mathbf{R}^{2}, \widehat{r}\right)$. Consider the Banach space $\mathcal{Y}$ of functions $\psi(\cdot): \mathbf{R} \times \mathbf{R} \times[0, T] \rightarrow \mathbf{R}$ such that

- $\psi(z, y, t)$ is continuous in $(y, t)$ given $z$

- the derivative $\frac{\partial \psi}{\partial z}(z, y, t)$ is continuous in $z$ for a.e. $(y, t)$;

- the following estimate is satisfied:

$$
\begin{aligned}
\|\psi(\cdot)\| \mathcal{Y} \triangleq \operatorname{ess}_{\sup }(|\psi(z, y, t)| & \left.+\left|\frac{\partial \psi}{\partial z}(z, y, t)\right|\right) \\
& +\sup _{t}\|\psi(\cdot, t)\|_{\mathcal{H}}+\left(\int_{0}^{T}\|\psi(\cdot, t)\|_{\mathcal{H}^{1}}^{2}\right)^{1 / 2}<+\infty
\end{aligned}
$$

In particular, if $\psi(\cdot) \in \mathcal{Y}$ then $\frac{\partial \psi}{\partial z}(z, y, t) \in L_{2}([0, T], \mathcal{H})$ and $\frac{\partial \psi}{\partial y}(z, y, t) \in L_{2}([0, T], \mathcal{H})$. 
Let $D \triangleq(0,+\infty) \times(0,+\infty), Q \triangleq D \times[0, T]$.

For $k>0$, let $\mathcal{Y}_{1}(k)$ denotes the class of all functions $G=G(x, v, t): Q \rightarrow \mathbf{R}$ such that $G(x, v, t)=\psi(k \log x, \log y, t)$, where $\psi(\cdot) \in \mathcal{Y}$.

Let $\mathcal{Y}_{0}(k)$ denotes the class of all functions $H=H(x, v, t): Q \rightarrow \mathbf{R}$ such that there exists a function $G(\cdot) \in \mathcal{Y}_{1}(k)$ such that $H(x, v, t)=G(x, v, t)+H_{B S}(x, v, t, k)$.

Let $\varphi_{1}(x, k) \triangleq(k-x)^{+}$, and let $\varphi_{2}(x, \tilde{k}) \triangleq(x-k)^{+}$.

Theorem 2.1 Assume that for any $K \in \mathcal{K}, \tilde{K}=e^{-r T} K, \varphi \equiv \varphi_{j}, j=1,2$, there exists a function $H(\cdot, \tilde{K})=H_{j}(\cdot, \tilde{K}) \in \mathcal{Y}_{0}(\tilde{K})$ that satisfies

$$
\left\{\begin{array}{l}
\frac{\partial H}{\partial t}(x, v, t, \tilde{K})+\frac{1}{2} x^{2} v \frac{\partial^{2} H}{\partial x^{2}}(x, v, t, \tilde{K})+\frac{1}{2} \widehat{\sigma}^{2} v^{2} \frac{\partial^{2} H}{\partial v^{2}}(x, v, t, \tilde{K})=0 \\
H(x, v, T, \tilde{K})=\varphi(x, \tilde{K}) \\
H(\tilde{K}, v, t, \tilde{K})=\tilde{H}_{B S}(\tilde{K}, v, t, \tilde{K}) \\
\left.\frac{\partial H}{\partial x}(x, v, t, \tilde{K})\right|_{x=\widehat{K}}=\left.\frac{\partial \tilde{H}_{B S}}{\partial x}(x, v, t, \tilde{K})\right|_{x=\widehat{K}} .
\end{array}\right.
$$

Then conditions (A1)-(A3) are satisfied for the market model with $\tilde{P}_{j}(t, \tilde{K}) \triangleq$ $H(\tilde{S}(t), v(t), t, \tilde{K})=H_{j}(\tilde{S}(t), v(t), t, \tilde{K})$ (in particular, the market is arbitrage free).

Remark. We mean that $(2.8)$ is satisfied for $H(\cdot, \tilde{K}) \in \mathcal{Y}_{0}(\tilde{K})$ in generalized sense as equality of generalized functions; the properties of $\mathcal{Y}_{0}(\tilde{K})$ are sufficient for correctness of all equations in (2.8).

Let $K \in \mathcal{K}$ be given, $\tilde{K}=e^{-r T} K$. Set

$$
\begin{gathered}
\varphi_{0}(x, v, t)=\frac{x}{\sqrt{2 \pi}} e^{-\frac{d_{+}(x, v, t, \tilde{K})^{2}}{2}}\left(\left[-\frac{\log x-\log \tilde{K}}{2 v \sqrt{(T-t) v}}+\frac{\sqrt{(T-t)}}{4 \sqrt{v}}\right]^{2}+\frac{3}{2} \frac{\log x-\log \tilde{K}}{2 v^{2} \sqrt{(T-t) v}}-\frac{\sqrt{(T-t)}}{8 v \sqrt{v}}\right) \\
-\frac{\tilde{K}}{\sqrt{2 \pi}} e^{-\frac{d_{-}(x, v, t, \tilde{K})^{2}}{2}}\left(\left[-\frac{\log x-\log \tilde{K}}{2 v \sqrt{(T-t) v}}-\frac{\sqrt{(T-t)}}{4 \sqrt{v}}\right]^{2}+\frac{3}{2} \frac{\log x-\log \tilde{K}}{2 v^{2} \sqrt{(T-t) v}}+\frac{\sqrt{(T-t)}}{8 v \sqrt{v}}\right), \\
\varphi(x, v, t) \triangleq \frac{1}{2} \widehat{\sigma}^{2} v^{2} \varphi_{0}(x, v, t),
\end{gathered}
$$

where $d_{+}(\cdot)$ and $d_{-}(\cdot)$ are defined by (2.5).

Set

$$
\mathbf{f}(z, v, t) \triangleq \varphi\left(\tilde{K} e^{z}, v, t\right)
$$

Set $I_{1} \triangleq(-\infty, 0), I_{2} \triangleq(0,+\infty)$

$$
\mathbf{F}_{m}(\omega, v, t) \triangleq \frac{1}{\sqrt{2 \pi}} \int_{I_{m}} e^{-i \omega z} \mathbf{f}(z, v, t) d z, \quad m=1,2
$$


Further, let

$$
\mathbf{U}_{m}(\omega, v, t) \triangleq \mathbf{E} \int_{t}^{T} \exp \left\{\frac{i \omega-\omega^{2}}{2} \int_{t}^{s} \mathbf{v}^{v, t}(q) d q\right\} \mathbf{F}_{m}\left(\omega, \mathbf{v}^{v, t}(s), s\right) d s,
$$

where $\mathbf{v}^{v, t}(s)$ is the solution of the Ito's equation (2.3) for the volatility process given the initial condition $\mathbf{v}^{v, t}(t)=v$, i.e.

$$
\left\{\begin{array}{l}
d_{s} \mathbf{v}^{v, t}(s)=\widehat{\sigma} \mathbf{v}^{v, t}(s) d \widehat{w}(s), \quad s>t \\
\mathbf{v}^{v, t}(t)=v
\end{array}\right.
$$

(Remind that $\widehat{a}=0$ in (2.3) by assumptions). Let

$$
\mathbf{u}(z, v, t) \triangleq \frac{1}{\sqrt{2 \pi}} \int_{\mathbf{R}} e^{i \omega z} \mathbf{U}_{m}(\omega, v, t) d z, \quad z \in I_{m}
$$

Theorem 2.2 Let $K \in \mathcal{K}, \tilde{K}=e^{-r T} K$ be given, and let $H(\cdot)=H_{j}(\cdot)$ be defined as

$$
H(x, v, t, \tilde{K}) \triangleq H_{B S, j}(x, v, t, \tilde{K})+\mathbf{u}\left(\log \frac{x}{\tilde{K}}, v, t\right) .
$$

Then $H(\cdot, \tilde{K}) \in \mathcal{Y}(\tilde{K})$ and it is the unique solution of (2.8) for $\varphi \equiv \varphi_{j}, j=1,2$ at this class; the integrals in (2.13) are defined as the Fourier transforms of functions that are square integrable as functions of $\omega$.

Remark. The function $\mathbf{u}(\cdot)$ does not depend on $j=1,2$; i.e., the correction for the Black-Scholes formula generated by this model is the same for call and put options.

\section{Proofs}

Proof of Proposition 2.1. Consider options with different strike prices $K \in \mathcal{K}$ as risky assets with prices $S_{K, j}(t)=P_{j}(t, K)$. Set $\tilde{S}_{K, j}(t) \triangleq e^{-r t} S_{K, j}(t)$. We have $P_{j}(t, K)=$ $e^{r t} \tilde{P}_{j}(t, \tilde{K}) \triangleq \tilde{H}_{B S, j}(\tilde{S}(t), v(t), t, \tilde{K})$. Then

$$
\begin{aligned}
& d \tilde{S}_{K, j}(t)+\frac{\partial \tilde{H}_{B S, j}}{\partial x}(\tilde{S}(t), v(t), t, \tilde{K}) d \tilde{S}(t)+\frac{\partial \tilde{H}_{B S, j}}{\partial v}(\tilde{S}(t), v(t), t, \tilde{K}) d v(t) \\
& +\left(\frac{\partial \tilde{H}_{B S, j}}{\partial t}(\tilde{S}(t), v(t), t, \tilde{K})\right. \\
& \left.+\frac{1}{2} x^{2} v \frac{\partial^{2} \tilde{H}_{B S, j}}{\partial x^{2}}(\tilde{S}(t), v(t), t, \tilde{K})+\frac{1}{2} \widehat{\sigma}^{2} v^{2} \frac{\partial^{2} \tilde{H}_{B S, j}}{\partial v^{2}}(\tilde{S}(t), v(t), t, \tilde{K})\right) d t \\
& =\frac{\partial \tilde{H}_{B S, j}}{\partial x}(\tilde{S}(t), v(t), t, \tilde{K}) d \tilde{S}(t) \\
& +\frac{\partial \tilde{H}_{B S, j}}{\partial v}(\tilde{S}(t), v(t), t, \tilde{K}) d v(t)+\frac{1}{2} \widehat{\sigma}^{2} v^{2} \frac{\partial^{2} \tilde{H}_{B S, j}}{\partial v^{2}}(\tilde{S}(t), v(t), t, \tilde{K}) d t
\end{aligned}
$$


Let $N \geq 2$. Let $K_{i} \in \mathcal{K}, \tilde{K}_{i}=e^{-r T} K_{i}, i=1,2, K_{1} \neq K_{2}$, let $j \in\{1,2\}$ be given, and let $X(t)$ be the wealth defined as

$$
X(t)=\beta(t) B(t)+\gamma_{0}(t) S(t)+\sum_{i=1}^{2} \gamma_{i, j}(t) P_{j}\left(t, K_{i}\right)
$$

for an admissible self-financing strategy $(\beta(t), \gamma(t))$ with $\gamma(t)=\left(\gamma_{0}(t), \gamma_{1, j}(t), \gamma_{2, j}(t)\right)$, such that $\gamma(\cdot) \neq 0$ and

$$
\begin{aligned}
& \gamma_{0}(t)=-\gamma_{1, j}(t) \frac{\partial \tilde{H}_{B S, j}}{\partial x}\left(\tilde{S}(t), v(t), t, \tilde{K}_{1}\right)-\gamma_{2, j}(t) \frac{\partial \tilde{H}_{B S, j}}{\partial x}\left(\tilde{S}(t), v(t), t, \tilde{K}_{2}\right), \\
& \gamma_{1, j}(t) \frac{\partial \tilde{H}_{B S, j}}{\partial v}\left(\tilde{S}(t), v(t), t, \tilde{K}_{1}\right)+\gamma_{2, j}(t) \frac{\partial \tilde{H}_{B S, j}}{\partial v}\left(\tilde{S}(t), v(t), t, \tilde{K}_{2}\right)=0 .
\end{aligned}
$$

(Clearly, such a strategy exists). Then

$$
d \tilde{X}(t)=\gamma_{0}(t) d \tilde{S}(t)+\sum_{i=1}^{2} \gamma_{i, j}(t) d \tilde{S}_{K_{i}}(t)=\xi(t) d t,
$$

where

$$
\xi(t)=\frac{1}{2} \gamma_{1, j}(t) \widehat{\sigma}^{2} v^{2} \frac{\partial^{2} \tilde{H}_{B S, j}}{\partial v^{2}}\left(\tilde{S}(t), v(t), t, \tilde{K}_{1}\right)+\frac{1}{2} \gamma_{2, j}(t) \widehat{\sigma}^{2} v^{2} \frac{\partial^{2} \tilde{H}_{B S, j}}{\partial v^{2}}\left(\tilde{S}(t), v(t), t, \tilde{K}_{2}\right) .
$$

We have that $\mathbf{P}(\xi(t) \neq 0)>0$. That means that arbitrage possibility does exist for $N \geq 2$.

Let $N=1$, and let $K \in \mathcal{K}, \tilde{K}=e^{-r T} K$. Let $X(t)$ be the wealth defined as

$$
X(t)=\beta(t) B(t)+\gamma_{0}(t) S(t)+\sum_{j=1}^{2} \gamma_{j}(t) P_{j}(t, K) .
$$

for an admissible self-financing strategy $(\beta(t), \gamma(t))$ with $\gamma(t)=\left(\gamma_{0}(t), \gamma_{j}(t), \gamma_{j}(t)\right)$. By (2.4), it follows that

$$
\begin{aligned}
& \frac{\partial \tilde{H}_{B S, 1}}{\partial v}(\tilde{S}(t), v(t), t, \tilde{K}) \equiv \frac{\partial \tilde{H}_{B S, 2}}{\partial v}(\tilde{S}(t), v(t), t, \tilde{K}), \\
& \frac{\partial^{2} \tilde{H}_{B S, 1}}{\partial v^{2}}(\tilde{S}(t), v(t), t, \tilde{K}) \equiv \frac{\partial^{2} \tilde{H}_{B S, 2}}{\partial v^{2}}(\tilde{S}(t), v(t), t, \tilde{K}) .
\end{aligned}
$$

Then there exist random $\mathcal{F}_{t}$-adapted processes $a_{i}(t)$ from $L^{2}(\Omega \times[0, T])$ such that

$$
d \tilde{X}(t)=\left[\alpha_{0}(t) \gamma_{0}(t)+\alpha_{1}(t) \gamma_{1}(t)+\alpha_{2} \gamma_{2}(t)\right] d \tilde{S}(t)+\left[\gamma_{1}(t)+\gamma_{2}(t)\left[\alpha_{2} d v(t)+\alpha_{4} d t\right]\right.
$$

It follows from independency of $w(\cdot)$ and $\widehat{w}(\cdot)$ that the market is arbitrage free for $N=1$. 
Proof of Theorem 2.1. Let $K \in \mathcal{K}, \tilde{K}=e^{-r T} K, S_{K, j}(t)=P_{j}(t, K), \tilde{S}_{K, j}(t) \triangleq$ $e^{-r t} S_{K, j}(t)$ again. We have $P_{j}(t, K)=e^{r t} \tilde{P}_{j}(t, \tilde{K}) \triangleq \tilde{H}_{j}(\tilde{S}(t), v(t), t, \tilde{K})$. Then

$$
\begin{aligned}
& d \tilde{S}_{K, j}(t)+\frac{\partial \tilde{H}_{j}}{\partial x}(\tilde{S}(t), v(t), t, \tilde{K}) d \tilde{S}(t)+\frac{\partial \tilde{H}_{j}}{\partial v}(\tilde{S}(t), v(t), t, \tilde{K}) d v(t) \\
& +\left(\frac{\partial \tilde{H}_{j}}{\partial t}(\tilde{S}(t), v(t), t, \tilde{K})+\frac{1}{2} x^{2} v \frac{\partial^{2} \tilde{H}_{j}}{\partial x^{2}}(\tilde{S}(t), v(t), t, \tilde{K})+\frac{1}{2} \widehat{\sigma}^{2} v^{2} \frac{\partial^{2} \tilde{H}_{j}}{\partial v^{2}}(\tilde{S}(t), v(t), t, \tilde{K})\right) d t \\
& =\frac{\partial \tilde{H}_{j}}{\partial x}(\tilde{S}(t), v(t), t, \tilde{K}) d \tilde{S}(t)+\frac{\partial \tilde{H}_{j}}{\partial v}(\tilde{S}(t), v(t), t, \tilde{K}) d v(t) .
\end{aligned}
$$

Let $K_{i} \in \mathcal{K}$, and let $X(t)$ be the wealth defined for some admissible self-financing strategy $(\beta(t), \gamma(t))$ as

$$
X(t)=\beta(t) B(t)+\gamma_{0}(t) S(t)+\sum_{j=1}^{2} \sum_{i=1}^{N} \gamma_{i, j}(t) P_{j}\left(t, K_{i}\right)
$$

Then

$$
d \tilde{X}(t)=\gamma_{0}(t) d \tilde{S}(t)+\sum_{j=1}^{2} \sum_{i=1}^{N} \gamma_{i, j}(t) d \tilde{S}_{K_{i}}(t) .
$$

Clearly, there exist random $\mathcal{F}_{t}$-adapted processes $c_{0}(t)$ and $c_{1}(t)$ from $L^{2}(\Omega \times[0, T])$ such that

$$
d \tilde{X}(t)=c_{0}(t) d \tilde{S}(t)+c_{1}(t) d v(t)
$$

It follows from independency of $w(\cdot)$ and $\widehat{w}(\cdot)$ that the market is arbitrage free.

Remark. Let $K_{i} \in \mathcal{K}, i=1,2$. The market with assets $\left(B(t), S(t), S_{K_{1}, 1}(t)\right)$ has two risky assets and two Brownian motions, and this market is arbitrage free. Assume that the corresponding function $H$ in Theorem 2.1 is such that $\frac{\partial H}{\partial v}\left(\tilde{S}(t), v(t), t, \tilde{K}_{1}\right) \neq 0$. Then the absence of arbitrage can be illustrated as

$$
\begin{aligned}
& d \tilde{S}_{K_{2}}(t)=\frac{\partial H}{\partial x}\left(\tilde{S}(t), v(t), t, \tilde{K}_{2}\right) d S(t)+\frac{\partial H}{\partial v}\left(\tilde{S}(t), v(t), t, \tilde{K}_{2}\right) d v(t) \\
& =\frac{\partial H}{\partial x}\left(\tilde{S}(t), v(t), t, \tilde{K}_{2}\right) d \tilde{S}(t) \\
& +\frac{\partial H}{\partial v}\left(\tilde{S}(t), v(t), t, \tilde{K}_{2}\right)\left[\frac{\partial H}{\partial v}\left(\tilde{S}(t), v(t), t, \tilde{K}_{1}\right)\right]^{-1}\left[d \tilde{S}_{K_{1}}(t)-\frac{\partial H}{\partial v}\left(\tilde{S}(t), v(t), t, \tilde{K}_{1}\right) d \tilde{S}(t)\right] .
\end{aligned}
$$

By (3.1), it follows that for any strategy for the market with assets $\left(B(t), S(t), S_{K_{1}, 1}(t)\right.$, $\left.S_{K_{2}, 1}(t)\right)$, there exists a strategy using assets $\left(B(t), S(t), S_{K_{1}, 1}(t)\right)$ only with the same wealth. Thus, adding options with different strike prices does not lead to arbitrage. 
Proof of Theorem 2.9. Instead of solving the problem (2.8), we shall solve the problem

$$
\left\{\begin{array}{l}
\frac{\partial G}{\partial t}(x, v, t)+\frac{1}{2} x^{2} v \frac{\partial^{2} G}{\partial x^{2}}(x, v, t)+\frac{1}{2} \widehat{\sigma}^{2} v^{2} \frac{\partial^{2} G}{\partial v^{2}}(x, v, t)=\phi(x, v, t), \\
G(x, v, T)=0 \\
G(\tilde{K}, v, t)=0 \\
\left.\frac{\partial G}{\partial x}(x, v, t)\right|_{x=\widehat{K}}=0
\end{array}\right.
$$

given function $\phi: Q \rightarrow \mathbf{R}$. Clearly, the desired solution $H(x, v, t, \tilde{K})$ can be found as $H(x, v, t, \tilde{K})=\tilde{H}_{B S}(x, v, t, \tilde{K})+G(x, v, t)$ if $\phi(x, v, t)=\frac{1}{2} \widehat{\sigma}^{2} v^{2} \frac{\partial^{2} \tilde{H}_{B S}}{\partial v^{2}}(x, v, t)$.

Introduce the Banach space $\mathcal{X}$ of functions $\phi: Q \rightarrow \mathbf{R}$ such that

$$
\begin{aligned}
\|\phi(\cdot)\|_{\mathcal{X}} & \triangleq \sup _{(v, t) \in Q} v^{-1 / 2}\left(\int_{\mathbf{R}}|f(z, v, t)| d z+\left[\int_{\mathbf{R}}|f(z, v, t)|^{2} d z\right]^{1 / 2}\right)<+\infty \\
& \text { where } f(z, v, t) \triangleq \phi\left(\tilde{K} e^{z}, v, t\right) .
\end{aligned}
$$

Let $j \in\{1,2\}$ and $\tilde{K}$ be fixed. Let $D_{1}(\tilde{K}) \triangleq\{(x, v) \in D: \quad x<\tilde{K}\}, D_{2}(\tilde{K}) \triangleq$ $\{(x, v) \in D: \quad x>\tilde{K}\}$. Let $Q_{m}(\tilde{K}) \triangleq D_{m}(\tilde{K}) \times[0, T], m=1,2$. Let $\mathcal{Y}_{j, m}(\tilde{K})$ denotes the class of functions $u=u(x, v, t): Q_{m}(K) \rightarrow \mathbf{R}$ such that $u(\cdot)=\left.\tilde{u}(\cdot)\right|_{Q_{m}(K)}$, where $\tilde{u}(\cdot) \in \mathcal{Y}_{j}(\tilde{K}), j=1,2$.

Instead of solving the problem (2.8), we shall investigate the pair of problems in $Q_{m}(\tilde{K})$, and $m=1,2$. Clearly, it suffices to prove the existence of a solution $G(\cdot) \in$ $\mathcal{Y}_{1, m}(\widehat{r}, \tilde{K})$ of the problem (3.2) in $Q_{m}(\tilde{K}), m=1,2$. (Recall that $\left.\tilde{K}=e^{-r T} K\right)$.

Let $\phi \in \mathcal{X}$,

$$
u(z, v, t) \triangleq G(x, v, t), \quad f(z, v, t) \triangleq \phi(x, v, t), \quad z \in \mathbf{R}, \quad x=\tilde{K} e^{z}
$$

Formally,

$$
\frac{\partial G}{\partial x}(x, v, t)=\frac{1}{x} \frac{\partial u}{\partial z}(z, v, t), \quad \frac{\partial^{2} G}{\partial x^{2}}(x, v, t)=\frac{1}{x^{2}} \frac{\partial^{2} u}{\partial z^{2}}(z, v, t)-\frac{1}{x^{2}} \frac{\partial u}{\partial z}(z, v, t) .
$$

Set $\widehat{Q}_{1} \triangleq\{(z, v, t): z \leq 0\}, \widehat{Q}_{2} \triangleq\{(z, v, t): z \geq 0\}$. Problem (3.2) can be rewritten for $u: \widehat{Q}_{m} \rightarrow \mathbf{R}, m=1,2$, as

$$
\left\{\begin{array}{l}
\frac{\partial u}{\partial t}(z, v, t)+\frac{v}{2} \frac{\partial^{2} u}{\partial z^{2}}(z, v, t)-\frac{v}{2} \frac{\partial u}{\partial z}(z, v, t)+\frac{1}{2} \widehat{\sigma}^{2} v^{2} \frac{\partial^{2} u}{\partial v^{2}}(z, v, t)=f(z, v, t), \\
u(z, v, T)=0 \\
u(0, v, t)=0 \\
\frac{\partial u}{\partial z}(0, v, t)=0 .
\end{array}\right.
$$


Assume that we can find the solution $u(z, v, t)$ of this problem in the class of functions that belong $L_{2}(\mathbf{R})$ as functions of $z$ and the following Fourier transform is defined:

$$
U(\omega, v, t) \triangleq \frac{1}{\sqrt{2 \pi}} \int_{I_{m}} e^{-i \omega z} u(z, v, t) d z, \quad \omega \in \mathbf{R}, \quad m=1,2 .
$$

Instead of (3.2), consider the problem for $U$

$$
\left\{\begin{array}{l}
\frac{\partial U}{\partial t}(\omega, v, t)+\left(-\omega^{2}-i \omega\right) \frac{v}{2} U(\omega, v, t)+\frac{1}{2} \widehat{\sigma}^{2} v^{2} \frac{\partial^{2} U}{\partial v^{2}}(\omega, v, t)=F(\omega, v, t) \\
U(\omega, v, T)=0
\end{array}\right.
$$

where

$$
F(\omega, v, t) \triangleq \frac{1}{\sqrt{2 \pi}} \int_{I_{m}} e^{-i \omega z} f(z, v, t) d z
$$

Proposition 3.1 There exist a constant $C_{1}>0$ such that for any $\phi(\cdot) \in \mathcal{X}$

$$
|F(\omega, v, t)| \leq C_{1} \sqrt{v}\|\phi(\cdot)\|_{\mathcal{X}} \quad \forall z \in \mathbf{R}, v>0, t \in[0, T], \omega \in \mathbf{R},
$$

where $F(\cdot)$ is defined by (3.7) and (3.3).

Proof. We have that $f(z, v, t)=c(z, v, t) \sqrt{v}$, where $\int_{I_{m}}|c(z, v, t)| d z \leq\|\phi(\cdot)\|_{\mathcal{X}}$. Clearly, the Fourier transform of $c(\cdot, v, t)$ is uniformly bounded.

Further, the solution of (3.6) given $\omega \in \mathbf{R}$ exists and can be expressed as

$$
U(\omega, v, t)=\mathbf{E} \int_{t}^{T} \exp \left\{\frac{-\omega^{2}+i \omega}{2} \int_{t}^{s} \mathbf{v}^{v, t}(q) d q\right\} F\left(\omega, \mathbf{v}^{v, t}(s), s\right) d s,
$$

where $\mathbf{v}^{v, t}(s)$ is the solution of the Ito's equation (2.12).

Lemma 3.1 There exist a constant $C>0$ such that

$$
\begin{aligned}
& \int_{-\infty}^{+\infty}\left(1+|\omega|^{2}\right)|U(\omega, v, t)|^{2} d \omega \leq C\|\phi(\cdot)\|_{\mathcal{X}} \quad \forall v>0, t \in[0, T], \\
& \int_{-\infty}^{+\infty}\left(1+|\omega|^{2}\right)^{p \delta / 2}|U(\omega, v, t)|^{p} d \omega \leq C\|\phi(\cdot)\|_{\mathcal{X}} \quad \forall p \geq 1, \delta \in[0,2-1 / p), v>0 .
\end{aligned}
$$

Proof. We have that

$$
\begin{aligned}
|U(\omega, v, t)| & \leq \frac{\tilde{K}}{2} \mathbf{E} \int_{t}^{T}\left|F\left(\omega, \mathbf{v}^{v, t}(s), s\right)\right| \exp \left\{\frac{-\omega^{2}}{2} \int_{t}^{s} \mathbf{v}^{v, t}(q) d q\right\} d s \\
& \leq \text { const }\|\phi(\cdot)\|_{\mathcal{X}} \mathbf{E} \int_{t}^{T} \sqrt{\mathbf{v}^{v, t}(s)} \exp \left\{\frac{-\omega^{2}}{2} \int_{t}^{s} \sqrt{\mathbf{v}^{v, t}(q)} d q\right\} d s \\
& \leq \text { const }\|\phi(\cdot)\|_{\mathcal{X}} \sqrt{T} \mathbf{E} \int_{t}^{T} \mathbf{v}^{v, t}(s) \exp \left\{\frac{-\omega^{2}+\alpha_{0}}{2} \int_{t}^{s} \mathbf{v}^{v, t}(q) d q\right\} d s \\
& =\text { const }\|\phi(\cdot)\|_{\mathcal{X}} \frac{2}{\omega^{2}} \mathbf{E}\left(1-\exp \left\{\frac{-\omega^{2}}{2} \int_{t}^{T} \mathbf{v}^{v, t}(q) d q\right\}\right)
\end{aligned}
$$


for all $v>0, t \in[0, T]$. This completes the proof.

Let

$$
\rho(y) \triangleq \frac{1}{1+e^{3 y}}, \quad y \in \mathbf{R} .
$$

Consider the Hilbert space $L_{2}(\mathbf{R}, \rho)$ with the weight $\rho(\cdot)$ that consist of complex valued functions $\eta: \mathbf{R} \rightarrow \mathbf{R}$ such that $\|\eta(\cdot)\|_{L_{2}(\mathbf{R}, \rho)} \triangleq\left(\int_{\mathbf{R}}|\eta(y)|^{2} \rho(y) d y\right)^{1 / 2}<+\infty$. The scalar product for that spaces is

$$
\left(\eta_{1}(\cdot), \eta_{2}(\cdot)\right)_{L_{2}(\mathbf{R}, \rho)} \triangleq \int_{\mathbf{R}} \bar{\eta}_{1}(y) \eta_{2}(y) \rho(y) d y .
$$

Let $\bar{X}$ be the Banach space of functions $\xi(\cdot): \mathbf{R} \times[0, T] \rightarrow \mathbf{R}$ such that $\xi(\cdot) \in$ $C\left([0, T] ; L_{2}(\mathbf{R}, \rho)\right)$ and

$$
\|\xi(\cdot)\|_{\bar{X}} \triangleq \sup _{t \in[0, T]}\|\xi(\cdot, t)\|_{L_{2}(\mathbf{R}, \rho)}+\left(\int_{0}^{T}\left\|\frac{\partial \xi}{\partial y}(\cdot, t)\right\|_{L_{2}(\mathbf{R}, \rho)}^{2} d t\right)^{1 / 2}<+\infty .
$$

Set

$$
\begin{aligned}
& \psi(z, y, t) \triangleq u\left(z, e^{y}, t\right)=G\left(\tilde{K} e^{z}, e^{y}, t\right), \\
& \Psi(\omega, y, t) \triangleq U\left(\omega, e^{y}, t\right), \\
& h(\omega, y, t) \triangleq F\left(\omega, e^{y}, t\right) .
\end{aligned}
$$

Lemma 3.2 There exist a constant $C>0$ such that

$$
\int_{\mathbf{R}}\|V(\omega, \cdot)\|_{\bar{X}}^{2} d \omega \leq C\|\phi(\cdot)\|_{\mathcal{X}}
$$

Proof. Set

$$
A \Psi=A(\omega, y, t) \Psi \triangleq \frac{\widehat{\sigma}^{2}}{2} \frac{\partial^{2} \Psi}{\partial y^{2}}(\omega, y, t)-\frac{\widehat{\sigma}^{2}}{2} \frac{\partial \Psi}{\partial y}(\omega, y, t), \quad g(\omega, y, t)=-\frac{e^{y} \omega^{2}}{2}-\frac{e^{y} i \omega}{2} .
$$

By the definitions, the function $\Psi$ satisfies

$$
\left\{\begin{array}{l}
\frac{\partial \Psi}{\partial t}(\omega, v, t)+A \Psi(\omega, y, t)+g(\omega, y, t) \Psi(\omega, y, t)=h(\omega, y, t) \\
\Psi(\omega, y, T)=0 .
\end{array}\right.
$$

Let the Hilbert space $\mathcal{L}$ be defined as $\mathcal{L} \triangleq L^{2}\left([0, T] ; L_{2}(\mathbf{R}, \rho)\right)$.

Let $\left\{h^{(j)}(\omega, y, t)\right\}_{j=1}^{+\infty}$ be a sequence of bounded smooth functions such that $h^{(j)} \rightarrow h$ in $\mathcal{L}=L^{2}\left([0, T] ; L_{2}(\mathbf{R}, \rho)\right)$ for any $\omega$. Let $\Psi=\Psi^{(j)}(\omega, \cdot)$ be the classical solution of (3.6) given $\omega$ for $h=h^{(j)}$. Clearly,

$$
\operatorname{Re}(\Psi(\omega, \cdot, t), g(\omega, \cdot, t) \Psi(\omega, \cdot, t))_{L_{2}(\mathbf{R}, \rho)}=\operatorname{Re} \int_{\mathbf{R}}|\Psi(\omega, y, t)|^{2} \frac{e^{y}\left(-\omega^{2}-i \omega\right)}{2} \rho(y) d y \leq 0 .
$$


Hence

$$
\begin{aligned}
& \frac{1}{2}\|\Psi(\omega, \cdot, s)\|_{L_{2}(\mathbf{R}, \rho)}^{2}-\frac{1}{2}\|\Psi(\omega, \cdot, T)\|_{L_{2}(\mathbf{R}, \rho)}^{2} \\
& =\operatorname{Re} \int_{s}^{T}(\Psi(\omega, \cdot, t), A \Psi(\omega, \cdot, t)+g(\omega, \cdot, t) \Psi(\omega, \cdot, t)+h(\omega, \cdot, t))_{L_{2}(\mathbf{R}, \rho)} d t \\
& =\operatorname{Re} \int_{s}^{T}\left\{-\frac{\widehat{\sigma}^{2}}{2}\left(\frac{\partial \Psi}{\partial y}(\omega, \cdot, t), \frac{\partial \Psi}{\partial y}(\omega, \cdot, t)\right)_{L_{2}(\mathbf{R}, \rho)}\right. \\
& -\left(\Psi(\omega, \cdot, t), \frac{\partial \rho}{\partial y}(\cdot) \frac{1}{\rho(\cdot)} \frac{\partial \Psi}{\partial y}(\omega, \cdot, t)\right)_{L_{2}(\mathbf{R}, \rho)}+\left(\Psi(\omega, \cdot, t), \frac{\partial \Psi}{\partial y}(\omega, \cdot, t)\right)_{L_{2}(\mathbf{R}, \rho)} \\
& \left.+(\Psi(\omega, \cdot, t), g(\omega, \cdot, t) \Psi(\omega, \cdot, t)+h(\cdot, t))_{L_{2}(\mathbf{R}, \rho)}\right\} d t \\
& \leq \int_{s}^{T}\left\{-\left(\frac{\widehat{\delta}^{2}}{2}-\varepsilon\right)\left\|\frac{\partial \Psi}{\partial y}(\omega, \cdot, t)\right\|_{L_{2}(\mathbf{R}, \rho)}^{2}\right. \\
& \left.+c_{1}(\varepsilon)\|\Psi(\omega, \cdot, t)\|_{L_{2}(\mathbf{R}, \rho)}^{2}+\|\Psi(\omega, \cdot, t)\|_{L_{2}(\mathbf{R}, \rho)}\|h(\omega, \cdot, t)\|_{L_{2}(\mathbf{R}, \rho)}\right\} d t \\
& \leq \int_{s}^{T}\left\{-\left(\frac{\widehat{\delta}^{2}}{2}-\varepsilon\right)\left\|\frac{\partial \Psi}{\partial y}(\omega, \cdot, t)\right\|_{L_{2}(\mathbf{R}, \rho)}^{2}\right. \\
& \left.+c_{2}(\varepsilon)\|\Psi(\omega, \cdot, t)\|_{L_{2}(\mathbf{R}, \rho)}^{2}+c_{3}(\varepsilon)\|h(\omega, \cdot, t)\|_{L_{2}(\mathbf{R}, \rho)}^{2}\right\} d t .
\end{aligned}
$$

Here $\varepsilon \in\left(0, \widehat{\delta}^{2} / 2\right)$ can be any, and $c_{k}(\varepsilon)$ depends only on $\varepsilon$; we have used the inequality $2 \alpha \beta \leq \varepsilon \alpha+\varepsilon^{-1} \beta(\forall \alpha, \beta, \varepsilon \in \mathbf{R}, \varepsilon>0)$. To derive (3.13), we have also used the equality

$$
\left(\Psi(\omega, \cdot, t), \frac{\partial \rho}{\partial y}(\cdot) \frac{\partial \Psi}{\partial y}(\omega, \cdot, t)\right)_{L_{2}(\mathbf{R})}=\left(\Psi(\omega, y, t), \frac{\partial \rho}{\partial y}(y) \frac{1}{\rho(y)} \frac{\partial \Psi}{\partial y}(\omega, y, t)\right)_{L_{2}(\mathbf{R}, \rho)} .
$$

Note that the function $\frac{\partial \rho}{\partial y}(y) \frac{1}{\rho(y)}$ is bounded.

By Bellman inequality, it follows from (3.13) that there exists a constant $C>0$ such that

$$
\sup _{t \in[0, T]}\|\Psi(\omega, \cdot, t)\|_{L_{2}(\mathbf{R}, \rho)}^{2}+\int_{0}^{T}\left\|\frac{\partial \Psi}{\partial y}(\omega, \cdot, t)\right\|_{L_{2}(\mathbf{R}, \rho)}^{2} d t \leq C\|h(\omega, \cdot)\|_{\mathcal{L}}^{2} \quad \forall \omega \in \mathbf{R}
$$

Hence there exists a constant $C>0$ such that

$$
\|\Psi(\omega, \cdot, t)\|_{\bar{X}} \leq C\|h(\omega, \cdot)\|_{\mathcal{L}} \quad \forall \omega \in \mathbf{R} .
$$

Remind that $\sup _{\omega, t}\|h(\omega, \cdot)\|_{\mathcal{L}} \leq$ const $\|\phi\|_{\mathcal{X}}$, and $\Psi=\Psi^{(j)}$ is the solution of (3.6) for $h=h^{(j)}$. Clearly, the sequence $\left\{\Psi^{(j)}\right\}_{j=1}^{+\infty}$ has the limit $\Psi \in \bar{X}$ that we shall call the solution of (3.6) for the original $h$ (this is in fact a generalized solution; the uniqueness of this solution follows from the linearity of the problem and from (3.15)).

Further, it follows from the estimation

$$
\int_{\mathbf{R}}|F(\omega, v, t)|^{2} d w=\text { const } \int_{\mathbf{R}}|f(z, v, t)|^{2} d z \leq \operatorname{const} v\|\phi(\cdot)\|_{\mathcal{X}}^{2}
$$


that

$$
\int_{\mathbf{R}}\|h(\omega, \cdot)\|_{\mathcal{L}}^{2} d \omega=\int_{\mathbf{R}} d \omega \int_{0}^{T}\|h(\omega, \cdot, t)\|_{L_{2}(\mathbf{R}, \rho)}^{2} d t \leq \operatorname{const}\|\phi(\cdot)\|_{\mathcal{X}}^{2}
$$

This completes the proof of Lemma 3.11.

Remind that $\Psi(\omega, y, t)=U\left(\omega, e^{y}, t\right)$. Clearly,

$$
\begin{aligned}
& \frac{\partial \Psi}{\partial y}(\omega, y, t)=\frac{\partial U}{\partial y}\left(\omega, e^{y}, t\right) e^{y} \\
& \psi(z, y, t)=\frac{1}{\sqrt{2 \pi}} \int_{\mathbf{R}} e^{i \omega z} \Psi(z, y, t) d z \\
& \frac{\partial \psi}{\partial y}(z, y, t)=\frac{1}{\sqrt{2 \pi}} \int_{\mathbf{R}} e^{i \omega z} \frac{\partial \Psi}{\partial y}(z, y, t) d z
\end{aligned}
$$

By Lemma 3.11,

$$
\int_{0}^{T} d t \int_{I_{m}} d z \int_{\mathbf{R}} \rho(y)\left(\left|u\left(z, e^{y}, t\right)\right|+\left|\frac{\partial u}{\partial y}\left(z, e^{y}, t\right) e^{y}\right|\right)^{2} d z \leq \text { const }\|\phi(\cdot)\|^{2} .
$$

Further, it follows from Lemma 3.1 that

$$
\sup _{t \in[0, T]} \int_{\mathbf{R}} e^{-|y|} d y \int_{I_{m}}\left(\left|u\left(z, e^{y}, t\right)\right|+\left|\frac{\partial u}{\partial z}\left(z, e^{y}, t\right)\right|\right)^{2} d z \leq \text { const }\|\phi(\cdot)\|_{\mathcal{X}}^{2} .
$$

Lemma 3.3 For any $\phi \in \mathcal{X}$, the derivative $\partial u(z, v, t) / \partial z$ is continuous in $z$ for a.e. $v, t$, and $\operatorname{ess}_{\sup }\left|\frac{\partial \psi}{\partial z}(z, y, t)\right| \leq$ const $\|\phi(\cdot)\|_{\mathcal{X}}$.

Proof. Remind that our selection of the version of the Fourier transforms means that $u(\cdot, v, t)$ is extended by zero from $I_{m}$ (or $u(\cdot)$ is extended by zero from $\widehat{Q}_{m}$ to $\widehat{Q}_{3-m}$, $m=1,2$, where $\left.\widehat{Q}_{1} \triangleq\{(z, v, t): z \leq 0\}, \widehat{Q}_{2} \triangleq\{(z, v, t): z \geq 0\}\right)$. By Lemma 3.1,

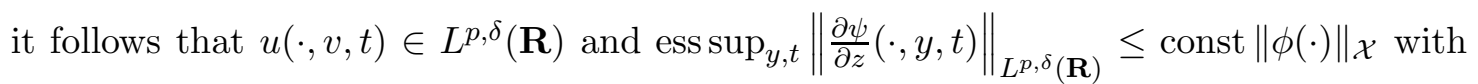
$p=3$ and $\delta \in\left(\frac{4}{3}, \frac{5}{3}\right)$, where $L^{p, s}(\mathbf{R})$ is the space described in Adams (1975), p. 220: it is the image of $L^{p}(\mathbf{R})$ under the linear mapping $J^{\delta}$ such that $\left.J^{s} u=\mathbf{F}^{-1}\left(1+|\cdot|^{2}\right)^{-\delta / 2} \mathbf{F} u\right)$, where $\mathbf{F}$ is the Fourier transform. By imbedding Theorems 7.63(g) and 7.57(c) from Adams (1975), it follows that the derivatives $\partial u(z, v, t) / \partial z$ are continuous for a.e. $v, t$ and

$$
\underset{y, t}{\operatorname{ess} \sup }\left(|\psi(z, y, t)|+\left|\frac{\partial \psi}{\partial z}(z, y, t)\right|\right) \leq \operatorname{const}\|\phi(\cdot)\|_{\mathcal{X}}
$$

Lemma 3.4 For any $\phi \in \mathcal{X}$, the function $u(z, v, t)$ is continuous in $t$ for a.e. $z, t$. 
Proof. It follows from (3.8) that $U(\omega, v, t)$ is continuous in $t$ for all $\omega, v$. Further, it follows from Lemma 3.1 that $\|U(\cdot, v, t)\|_{L_{1}(\mathbf{R})} \leq$ const $<+\infty$. By Dominated Convergency Theorem it follows continuity of the Fourier transform.

We can summarize the results of Lemmas 3.1 3.4 in the following theorem.

Theorem 3.1 For any $\phi \in \mathcal{X}$, the function $\psi(z, y, t)$, defined in (3.10) belongs $\mathcal{Y}$, and there exists a constant $C>0$ such that

$$
\|\psi(\cdot)\|_{\mathcal{Y}} \leq \mathrm{const}\|\phi(\cdot)\|_{\mathcal{X}} \quad \forall \phi \in \mathcal{X}
$$

Proposition 3.2 In (2.9), $\varphi(x, v, t)=\frac{1}{2} \widehat{\sigma}^{2} v^{2} \frac{\partial^{2} \tilde{H}_{B S}}{\partial v^{2}}(x, v, t)$, and $\varphi(\cdot) \in \mathcal{X}$.

Proof. By (2.4), it follows that

$$
\begin{aligned}
\frac{\partial \tilde{H}_{B S}}{\partial v}(x, v, t, \tilde{K})= & \frac{x}{\sqrt{2 \pi}} e^{-\frac{d_{+}(x, v, t, \tilde{K})^{2}}{2}} \frac{\partial d_{+}}{\partial v}(x, v, t, \tilde{K})-\frac{\tilde{K}}{\sqrt{2 \pi}} e^{-\frac{d_{-}(x, v, t, \tilde{K})^{2}}{2}} \frac{\partial \bar{d}}{\partial v}(v, t) \\
= & \frac{x}{\sqrt{2 \pi}} e^{-\frac{d_{+}(x, v, t, \tilde{K})^{2}}{2}}\left[-\frac{\log x-\log \tilde{K}}{2 v \sqrt{(T-t) v}}+\frac{\sqrt{(T-t)}}{4 \sqrt{v}}\right] \\
& -\frac{\tilde{K}}{\sqrt{2 \pi}} e^{-\frac{d_{-}(x, v, t, \tilde{K})^{2}}{2}}\left[-\frac{\log x-\log \tilde{K}}{2 v \sqrt{(T-t) v}}-\frac{\sqrt{(T-t)}}{4 \sqrt{v}}\right] .
\end{aligned}
$$

Then

$$
\begin{aligned}
& \frac{\partial^{2} \tilde{H}_{B S}}{\partial v^{2}}(x, v, t, \tilde{K}) \\
& =\frac{x}{\sqrt{2 \pi}} e^{-\frac{d_{+}(x, v, t, \tilde{K})^{2}}{2}}\left(\left[-\frac{\log x-\log \tilde{K}}{2 v \sqrt{(T-t) v}}+\frac{\sqrt{(T-t)}}{4 \sqrt{v}}\right]^{2}+\frac{3}{2} \frac{\log x-\log \tilde{K}}{2 v^{2} \sqrt{(T-t) v}}-\frac{\sqrt{(T-t)}}{8 v \sqrt{v}}\right) \\
& \quad-\frac{\tilde{K}}{\sqrt{2 \pi}} e^{-\frac{d_{-}(x, v, t, \tilde{K})^{2}}{2}}\left(\left[-\frac{\log x-\log \tilde{K}}{2 v \sqrt{(T-t) v}}-\frac{\sqrt{(T-t)}}{4 \sqrt{v}}\right]^{2}+\frac{3}{2} \frac{\log x-\log \tilde{K}}{2 v^{2} \sqrt{(T-t) v}}+\frac{\sqrt{(T-t)}}{8 v \sqrt{v}}\right) .
\end{aligned}
$$

Clearly, $\varphi(\cdot)$ is continuous in $(0,+\infty) \times(0,+\infty) \times[0, T)$, and the limit of $\varphi(\cdot)$ is $\mathcal{O}\left(v^{1 / 2}\right)$ as $x \rightarrow 0, x \rightarrow \tilde{K}, x \rightarrow+\infty$ as well as $v \rightarrow 0, v \rightarrow+\infty$ and $t \rightarrow T$. The required summarability is ensured by properties of $e^{d_{ \pm}}$.

Now we are in the position to complete the proof of Theorem 2.2.

From Theorem 3.1, the existence of solution in Theorem 2.2 follows. The fact that the solution is unique in the given class follows from (3.18). The formula for $\mathbf{u}$ from (2.14) is in fact the formula derived above for $u$ with substituting $\phi(\cdot)=\varphi(\cdot)$.

\section{References}


Adams, R.A. (1975). Sobolev Spaces. Academic press.

Black, F. and M. Scholes (1972): The valuation of options contracts and test of market efficiency. Journal of Finance, 27, 399-417.

Black, F. and M. Scholes (1973): The pricing of options and corporate liabilities. Journal of Political Economics, 81, 637-659.

Christie, A. (1982): The stochastic behaviour of common stocks variances: values, leverage, and interest rate effects. Journal of Financial Economics, 10, 407-432.

Day, T.E. and C.M. Levis (1992): Stock market volatility and the information content of stock index options. Journal of Econometrics, 52, 267-287.

Derman, E., I. Kani, and J.Z. Zou (1996): The local volatility surface: unlocking the information in index option prices. Financial Analysts Journal 25-36.

Geman, H. and T. Ane (1996): Stochastic subordination. Risk, 9 (9), 145-149.

Dokuchaev, N.G. (1995): Probability distributions of Ito's processes: estimations for density functions and for conditional expectations of integral functionals. Theory of Probability and Its Applications, 39, iss. 4, 662-670.

Dokuchaev, N.G., and X.Y. Zhou (2001): Optimal investment strategies with bounded risks, general utilities, and goal achieving. Journal of Mathematical Economics, 35, iss.2, 289-309.

Dokuchaev N.G. (2002): Dynamic portfolio strategies: quantitative methods and empirical rules for incomplete information. Kluwer Academic Publishers, Boston.

Harrison, J.M., and S.R. Pliska (1981): Martingales and stochastic integrals in the theory of stochastic trading. Stochastic Processes and their Application, 11, 215-260.

Hauser, S. and B. Lauterbach (1997): The relative performance of five alternative warrant pricing models. Financial Analysts Journal, N1, 55-61.

Hull, J. and A. White (1987): The pricing of options on assets with stochastic volatilities. Journal of Finance, 42, 281-300.

Jarrow, R. (ed.) (1998): Volatility New Estimations Techniques for pricing Derivatives, Risk Books.

Johnson, H. and D. Shanno (1987): Option pricing when the variance is changing. Journal of Financial and Quantitative Analysis, 22, 143-151. 
Jouini, E. Market imperfection, equilibrium and arbitrage. (1996): Financial Mathematics (Lecture Notes in Mathematics, 1656), 247-307.

Masi, G.B., Yu.M. Kabanov, and W.J. Runggaldier (1994): Mean-variance hedging of options on stocks with Markov volatilities. Theory of Probability and Its Applications $39,172-182$.

Mayhew, S. (1995): Implied volatility. Financial Analysts Journal, iss. 4, 8-20.

Taylor, S.J. and X. Xu (1994): The magnitude of implied volatility smiles: theory and empirical evidence for exchange rates. Review of Future Markets, 13, 355-380. 